\title{
Minireview
}

Jone López-Erauskin, Isidre Ferrer, Elena Galea ${ }^{\mathrm{a}}$ and Aurora Pujol ${ }^{\mathrm{a}, \star}$

\section{Cyclophilin D as a potential target for antioxidants in neurodegeneration: the X-ALD case}

\begin{abstract}
X-linked adrenoleukodystrophy (X-ALD) is a severe inherited neurodegenerative disorder characterized by adrenal insufficiency and graded damage in the nervous system. Loss of function of the peroxisomal ABCD1 fatty-acid transporter, resulting in the accumulation of very long-chain fatty acids in organs and plasma, is the genetic cause. Treatment with a combination of antioxidants halts the axonal degeneration and locomotor impairment displayed by the animal model of X-ALD, and is a proof of concept that oxidative stress contributes to axonal damage. New evidence demonstrates that metabolic failure and the opening of the mitochondrial permeability transition pore orchestrated by cyclophilin D underlies oxidative stress-induced axonal degeneration. Thus, cyclophilin D could serve as a therapeutic target for the treatment of X-ALD and cyclophilin D-dependent neurodegenerative and non-neurodegenerative diseases.
\end{abstract}

Keywords: cyclophilin D; neurodegeneration; mitochondrial permeability transition pore; oxidative stress; very long chain fatty acids; X-linked adrenoleukodystrophy.

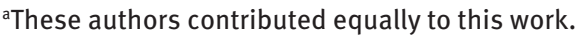
*Corresponding author: Aurora Pujol, Neurometabolic Diseases Laboratory, Bellvitge Biomedical Research Institute (IDIBELL), L' Hospitalet de Llobregat, E-08908 Barcelona, Spain; Center for Biomedical Research on Rare Diseases (CIBERER) U759, L' Hospitalet de Llobregat, E-08908 Barcelona, Spain; Institute of Neuropathology, Pathologic Anatomy Service, Bellvitge Biomedical Research Institute, IDIBELL-Hospital Universitari de Bellvitge, L' Hospitalet de Llobregat, E-08908 Barcelona, Spain; and Catalan Institution of Research and Advanced Studies (ICREA), E-08010 Barcelona, Spain, e-mail: apujol@idibell.cat Jone López-Erauskin: Neurometabolic Diseases Laboratory, Bellvitge Biomedical Research Institute (IDIBELL), L' Hospitalet de Llobregat, E-08908 Barcelona, Spain; Center for Biomedical Research on Rare Diseases (CIBERER) U759, L' Hospitalet de Llobregat, E-08908 Barcelona, Spain; and Institute of Neuropathology, Pathologic Anatomy Service, Bellvitge Biomedical Research Institute, IDIBELL-Hospital Universitari de Bellvitge, L' Hospitalet de Llobregat, E-08908 Barcelona, Spain Isidre Ferrer: Institute of Neuropathology, Pathologic Anatomy Service, Bellvitge Biomedical Research Institute, IDIBELLHospital Universitari de Bellvitge, L' Hospitalet de Llobregat,
}

E-08908 Barcelona, Spain; and Center for Biomedical Research on Neurodegenerative Diseases (CIBERNED), L' Hospitalet de Llobregat, E-08908 Barcelona, Spain

Elena Galea: Institute of Neuroscience, Universitat Autònoma de Barcelona, E-08193 Barcelona, Spain; and Catalan Institution of Research and Advanced Studies (ICREA), E-08010 Barcelona, Spain

\section{Introduction: general features of $X$-linked adrenoleukodystrophy and animal models of the disease}

X-linked adrenoleukodystrophy (X-ALD: McKusick no. 300100) is a rare neurometabolic disease characterized by progressive demyelination within the central nervous system (CNS), axonopathy in the spinal cord, and adrenal insufficiency. This inheritable disorder is caused by mutations in the $A B C D 1(A L D)$ gene located in Xq28. This encodes for the peroxisomal ABCD (ALD protein) transporter (Mosser et al., 1993), which is involved in importing very long-chain fatty acids (VLCFAs) and very long-chain fatty acid-Coenzyme A (VLCFA-CoA) esters into the peroxisome, where they are degraded by $\beta$-oxidation (Singh et al., 1984; Wanders et al., 1987; van Roermund et al., 2008; Fourcade et al., 2009). Defective function of the ABCD1 transporter leads to VLCFA accumulation in most organs and plasma, and elevated levels of VLCFAs are used as a biomarker for the biochemical diagnosis of the disease (Berger and Gartner, 2006; Moser et al., 2007). X-ALD is the most common monogenic leukodystrophy and peroxisomal disorder, occurring in at least 1 out of 16800 people (Bezman et al., 2001).

Four major disease variants have been described:

- Adrenomyeloneuropathy (AMN), a late-onset form presenting a slow progression and characterized by a peripheral neuropathy and distal axonopathy in the spinal cord. This phenotype is often, but not always, associated with axonal or demyelinating peripheral neuropathy and can affect both adult men and women. 
- AMN with cerebral demyelination or cAMN. This form mainly affects adult males and can turn into an inflammatory phenotype in many cases.

- The cerebral inflammatory demyelination form that affects boys, adolescents and adult males or cALD.

- An adrenal insufficiency (Addison's disease) that affects boys, adolescents and adult males, and presents a partial penetrance.

Patients suffering this last variant of X-ALD have a high risk of developing later cerebral demyelination or AMN (Laureti et al., 1996; Engelen et al., 2012). The cerebral inflammatory demyelinating forms of X-ALD are fatal if not treated by bone marrow transplant in the early stages of the disease (Shapiro et al., 2000; Peters et al., 2004).

Irrespective of its location or variant, a cardinal event of X-ALD is axonal damage. In variants affecting the brain, demyelination appears to be the main cause of severe axonal damage and neuronal death, whereas in AMN axonal damage in the spinal cord and peripheral nerves is the predominant feature, with demyelination as a secondary process (Powers et al., 2000; Ferrer et al., 2010).

It is well known that a murine model offers great advantages over postmortem patient materials, such as the possibility of carrying out a longitudinal characterization of the disease process, thus allowing the discrimination of causative events from epiphenomena. Three mouse models for X-ALD were independently generated following a classical strategy of knocking out the Abcd1 gene, which is located in the $\mathrm{X}$ chromosome of the mouse genome (Forss-Petter et al., 1997; Kobayashi et al., 1997; $\mathrm{Lu}$ et al., 1997). All three mouse models showed high levels of VLFCAs in the nervous tissue, adrenal glands and other organs as a representative signature, but in contrast, none of them displayed cerebral demyelination or inflammatory signs up to 6 months of age (ForssPetter et al., 1997; Kobayashi et al., 1997; Lu et al., 1997). However, an analysis of animals at 16 months of age with a pure $\mathrm{C} 57 \mathrm{BL} / 6 \mathrm{~J}$ genetic background showed histological signs of axonopathy in the sciatic nerves, and interestingly, at 20-22 months of age mice displayed microglia activation, astrocytosis, and axonal swelling and damage (Pujol et al., 2002, 2004). All these effects were concomitant with locomotor alterations and delays of motor nerve conduction velocities (Pujol et al., 2002; Ferrer et al., 2005). Thus, this mouse model resembles the characteristic axonopathy in the spinal cord of patients with pure AMN (Pujol et al., 2002), and it constitutes a good model for dissecting physiopathogenetic mechanisms. A second peroxisomal transporter and the closest homolog, the $A B C D 2$ protein, shares functions in the import of
C26:0, the main VLCFA accumulated in X-ALD (Pujol et al., 2004; Ferrer et al., 2005; Fourcade et al., 2009). The double knockout $A b c d 1 / A b c d 2$ displays a more severe and earlier onset axonopathy that facilitates preclinical testing (Pujol et al., 2004).

Although considerable efforts have been made in the development of efficient therapies against this fatal disease, pharmacological treatment options for X-ALD are scarce (reviewed in Berger et al., 2010). We and others have demonstrated that oxidative stress is a common feature in X-ALD patients (Vargas et al., 2004; Powers et al., 2005; Fourcade et al., 2010) as well as in the animal model, appearing very early on in the latter (Fourcade et al., 2008). These results have prompted us to explore antioxidant treatment as a therapeutic option, and to this end we recently performed preclinical trials with a cocktail of antioxidants containing $\alpha$-tocopherol, $\mathrm{N}$-acetylcysteine and $\alpha$-lipoic acid in $A b c d 1$-mice. The results were: - correction of oxidative damage to proteins and DNA;

- correction of metabolic deficits including levels of ATP, NADH and pyruvate kinase activity;

- reversal of axonal damage; and

- reversal of the locomotor disability provoked by the accumulation of VLCFA in the $A b c d 1 / A b c d 2$ null mutants (Galino et al., 2011; Lopez-Erauskin et al., 2011).

A Phase II clinical trial with these antioxidants is currently in progress (NCT01495260). However, the molecular mechanisms mediating the beneficial effect of the antioxidants remain unclear.

The purpose of this review is to highlight recent evidence about the molecular events occurring during the cellular damage triggered by oxidative stress and the role played by cyclophilin D (CypD). Moreover, using data obtained with antioxidant treatment we will discuss the potential of using CypD as a mechanistic action model of how antioxidants are able to prevent axonal neurodegeneration. CypD comes to center stage as an essential step in mitochondrial collapse in neurodegenerative diseases including X-ALD.

\section{Very long-chain fatty acids and oxidative stress}

Although patients with X-ALD, characterized by abnormal VLCFA accumulation, were first described at the beginning of the last century, the demonstration that VLCFA accumulation led to toxicity was only recently made. In 2008, our 
group and others reported that VLCFA accumulation was not an epiphenomenon but, rather, that VLCFA in excess was toxic (Fourcade et al., 2008; Hein et al., 2008). Using human fibroblasts we showed that VLCFA accumulation generates important levels of radical oxygen species (ROS), decreases reduced glutathione (GSH) levels, and induces inner mitochondrial membrane depolarization (Fourcade et al., 2008). In agreement with these data, parallel studies performed in rat primary neural cell cultures showed that VLCFAs are able to dissipate inner mitochondrial membrane potential and increase intracellular calcium levels in oligodendrocytes (Hein et al., 2008). Similarly, the treatment of VLCFAs increases ROS levels in the murine oligodendrocyte cell line 158N (Baarine et al., 2012a,b) and human neuronal cell line SK-NB-E (Zarrouk et al., 2012), besides inducing a decrease in GSH levels and the dissipation of the inner mitochondrial membrane potential (Baarine et al., 2012a,b; Zarrouk et al., 2012). Moreover, incubation of fibroblasts with C26:0 only provoked oxidative damage to proteins and lipids in X-ALD fibroblasts and not in control fibroblasts (Fourcade et al., 2008). In addition, the X-ALD fibroblasts present increased vulnerability to oxidative stress under depleted GSH conditions (Fourcade et al., 2008).

Oxidative damage is indeed the main etiopathogenic factor in X-ALD disease; it is observed very early on in the spinal cord of the mouse model and in fibroblasts from patients with X-ALD (Fourcade et al., 2008; Singh and Pujol, 2010; Lopez-Erauskin et al., 2011). This damage is characterized by an increase in the quantitative markers of protein lipoxidation (malondialdehyde-lysine), glycoxidation and lipoxidation (carboxyethyl-lysine), together with markers of the direct carbonylation of proteins (glutamic semialdehyde and aminoadipic semialdehyde) in the pre-symptomatic stages of the X-ALD mouse model (Fourcade et al., 2008). In accordance with these data, signs of oxidative modifications have also been described in postmortem X-ALD brains, as well as in plasma and blood cells (Vargas et al., 2004; Powers et al., 2005; Fourcade et al., 2010).

These in vivo observations support the surrogate use of skin-derived fibroblasts from patients as a successful in vitro model for the study of the molecular mechanisms of this disease instead of human CNS tissues, which are only accessible postmortem.

It is not clear how VLCFAs trigger oxidative stress, but it is worth noting that VLCFAs ( $\mathrm{C} \geq 22: 0)$ are usually constituents of complex lipids, such as gangliosides, phosphatidylcholine, and cholesterol ester fractions of brain myelin and of proteolipid protein. While the gangliosides of normal individuals lack VLCFAs (Garashi et al., 1976), the gangliosides in the brain of X-ALD patients contain 28-50\% VLCFAs. Their incorporation in lipid membranes may cause cell and organelle dysfunction (Powers and Moser, 1998). In fact, lysophosphatidylcholine fractions have been found to be particularly enriched with C26:0 (Hubbard et al., 2006). Moreover, an excess of lisophosphatidylcholine-C24:0 species produces inflammatory demyelination when injected in normal mice (Eichler et al., 2008).

\section{Oxidative stress and metabolic failure are intertwined in X-linked adrenoleukodystrophy}

Oxidative stress is a condition resulting from a redox imbalance that leads to an excess of ROS. Due to high instability, ROS react with and induce chemical changes in surrounding molecules. These changes are detrimental if the modified residues happen to be essential for the activity or turnover of the molecule (Wang et al., 2012). Macromolecules such as DNA, lipids and proteins, are highly vulnerable to ROS attack. Moreover, oxidative lesions can also transform lipid properties and induce mutations in mitochondrial and nuclear DNA, causing mitochondrial dysfunction and pathology (Pamplona and Barja, 2007).

In X-ALD, we have identified a common signature present in the spinal cord of mice and brains of cAMN and cALD patients. This reflects a metabolic-inflammatory interplay through IkB kinase and oxidative stress induced by excess VLCFAs (Schluter et al., 2012). Indeed, using a combination of proteomic approaches and mass spectrometry, we discovered that the absence of ABCD1 causes oxidative lesions in five key enzymes of glycolysis and the tricarboxylic acid cycle: aldolase A, phosphoglycerate kinase, pyruvate kinase, dihydrolipoamide dehydrogenase and aconitase (Galino et al., 2011). Metabolomic measurements of different substrates and products confirmed that the oxidation of these enzymes inhibits their catalytic activity, thus decreasing NADH and ATP levels. This, in turn, results in a syndrome of energetic deficiency (Galino et al., 2011). However, antioxidants halted the oxidative stress-induced metabolic alterations in an X-ALD mouse model, normalizing NADH, ATP, NADPH and GSH levels (Galino et al., 2011). These data lend support to the idea that there is metabolic derangement in the spinal cords of $A b c d r$ mice prior to the development of neurological symptoms. Based on the animal data, it is reasonable to propose that the energy failure produced by oxidative 
stress may be a physiopathogenic factor, at least in adult forms of X-ALD.

Nonetheless, oxidative stress is not an exclusive event of X-ALD; common neurodegenerative diseases such as Alzheimer's, Huntington's and Parkinson's also show redox imbalance, protein oxidation and energetic failure (Lin and Beal, 2006; Pratico, 2008; Stack et al., 2008; Zhou et al., 2008; Martinez et al., 2010).

Many of the aforementioned alterations reveal the high susceptibility of the CNS to oxidative damage, probably related to the large amount of highly peroxidizable polyunsaturated fatty acids in membranes, the presence of catecholamines prone to oxidation, elevated oxygen consumption, and the relatively poor expression of enzymatic antioxidant defenses (Lin and Beal, 2006; Naudi et al., 2011). Moreover, neurons are more dependent on mitochondria than on glycolysis for ATP production, which renders them particularly vulnerable to mitochondrial dysfunction (Thannickal and Fanburg, 2000).

\section{Oxidative stress and mitochondrial dysfunction}

Mitochondria orchestrate essential functions, including ROS, calcium homeostasis, and energy generation, as well as cell survival and death. For this reason, optimal functioning of mitochondria is required to preserve cell and organism viability. Mitochondria are highly susceptible to oxidative damage because they are the main producers of ROS as a byproduct of the electron transport chain. In the majority of neurometabolic disorders, mitochondrial dysfunction is a central marker of disease progression. Indeed, the accumulation of damaged mitochondria correlates with the development of the disease (Lin and Beal, 2006; Du and Yan, 2010; Fernandez-Checa et al., 2010; Blackstone and Chang, 2011).

The mitochondrial permeability transition pore $(\mathrm{mPTP})$ is a protein pore formed in theinner membrane of the mitochondria under certain pathological conditions, allowing the flux of solutes of up to $1500 \mathrm{Da}$ (Bernardi et al., 2006; Halestrap, 2009). This results in the dissipation of mitochondrial membrane potential and damage to the mitochondrial respiratory chain, thereby reducing ATP production and exacerbating mitochondrial dysfunction. Moreover, there is osmotic swelling that ruptures the outer mitochondrial membrane (Baines et al., 2005; Bernardi et al., 2006; Du and Yan, 2010). Overall, these phenomena irreversibly trigger cell death (Petronilli et al., 1994; Baines et al., 2005; Halestrap, 2005; Nakagawa et al., 2005).
Although the role of mPTP in pathological conditions has been widely established, the nature and identity of the molecular components of the pore are still under discussion, with CypD being the only component that appears to be essential (Baines et al., 2005; Nakagawa et al., 2005; Schinzel et al., 2005). CypD is an $18 \mathrm{kDa}$ peptidyl-prolylcis-trans-isomerase located in the mitochondrial matrix. In physiological conditions it plays an important role in protein folding due to its ability to catalyze the cis-trans isomerization of peptidylprolyl bonds (Schonbrunner et al., 1991). However, once certain pathological conditions are established, CypD is translocated to the inner mitochondrial membrane to induce the opening of the mPTP (Connern and Halestrap, 1994). The genetic abrogation of CypD prevents mPTP formation under oxidative stress and/or calcium overload (Baines et al., 2005), supporting the essential role of CypD in mPTP formation.

\section{The mitochondrial permeability transition pore in the physio- pathogenesis of X-linked adrenoleukodystrophy}

Many reports indicate that the formation of MPTP is a key factor in mitochondrial dysfunction and mitochondria-driven cell death (Halestrap, 2005; Du and Yan, 2010). These abnormalities are thus rescued by the addition of the CypD inhibitor CsA, or by the genetic blockade of CypD (Crompton et al., 1988; Halestrap and Davidson, 1990; Baines et al., 2005). Indeed, the genetic abrogation of CypD prevents mitochondrial dysfunction in many age-related neurodegenerative disease models, such as Alzheimer's disease (Du et al., 2008), Parkinson's disease (Gandhi et al., 2009; Thomas et al., 2011), amyotrophic lateral sclerosis (Karlsson et al., 2004; Martin et al., 2009; Martin, 2010) and multiple sclerosis (Forte et al., 2007).

ROS and calcium are potent inducers of mPTP opening (Baines et al., 2005; Bernardi et al., 2006; Du and Yan, 2010). ROS and calcium signaling are altered in X-ALD (Fourcade et al., 2008; Hein et al., 2008), therefore we sought to determine whether both factors were relevant to $\mathrm{X}$-ALD pathology. We recently reported that CypD is significantly increased in X-ALD mouse model spinal cords, in fibroblasts from X-ALD patients, and in the affected zones of brains from AMN patients (Lopez-Erauskin et al., 2012). This is reminiscent of the increased CypD expression reported in brains from patients with Alzheimer's disease 
(Du et al., 2008) and Huntington's disease (Shirendeb et al., 2011).

Interestingly, antioxidant treatment was able to reduce the high levels of CypD observed in fibroblasts from X-ALD patients and in spinal cords from X-ALD mice (LopezErauskin et al., 2012). Also, the induction of oxidative stress in control human fibroblasts in a glucose-free medium containing galactose, or with an excess of C26:0, causes an increase in CypD protein expression (Lopez-Erauskin et al., 2012). All in all, the evidence indicates that CypD expression depends on the redox state. Moreover, not only the expression but also the activity of CypD may be regulated by oxidative stress, since we detected oxidative modifications in CypD in X-ALD fibroblasts, which can be reversed by classical antioxidants both in vivo and in vitro (Lopez-Erauskin et al., 2012). In accordance with this, it has been suggested that CypD is a redox sensor (Linard et al., 2009; Nguyen et al., 2011). Linard and coworkers identified cysteine 203 in CypD as being a redox-sensitive residue, and reported a reduction in oxidative stress-induced CypD peptidylprolyl-cis-trans-isomerase activity, together with oxidative stress-induced CypD protein conformational changes (Linard et al., 2009). It was proposed that, once oxidative stress is triggered, the oxidation state of CypD induces conformational changes in its structure, promoting its translocation to the inner mitochondrial membrane via the MPTP opening, which eventually contributes to the necrotic death process.

Along these lines, the finding that CsA, the CypD inhibitor, prevented the galactose-induced cell death of X-ALD fibroblasts (Lopez-Erauskin et al., 2012) confirms that CypD mediates the damage brought about by oxidative stress in X-ALD.

Although the relevance of CypD in X-ALD will only be unequivocally confirmed by the genetic ablation of CypD, with all the evidence obtained thus far we may suggest that the oxidative stress-induced and CypD-dependent opening of mPTP is a central feature of X-ALD. We therefore propose that CypD is a key molecule in X-ALD physiopathology and is an important new target for therapeutic intervention.

\section{Concluding remarks}

\section{X-linked adrenoleukodystrophy is caused by the oxidative stress-initiated interplay of mitochondrial collapse and energetic failure}

Our working model is as follows (Figure 1A). ROS, generated by C26:0 accumulation, induce energetic failure by oxidizing glycolytic and tricarboxylic acid cycle enzymes, which leads to a decrease in NADH and ATP content. The parallel decrease of GSH, the most important antioxidant defense of the cell, will contribute to the oxidative stress. The increased ROS content further worsens mitochondrial dysfunction by opening the MPTP, which in turn is exacerbated by the translocation of oxidized and overexpressed CypD to the inner mitochondrial membrane. That is, oxidative stress causes cell death in X-ALD in association with severe mitochondrial dysfunction, leading to metabolic failure. As a word of caution, it should be stressed that the above conclusions refer to skin fibroblasts. A precise dissection of the role played by mitochondria dysfunction compared to other sources of free radicals needs to be undertaken in the different neural cell types of the nervous system.

\section{Cyclophilin D is a novel therapeutic target}

The inhibition of CypD overexpression and activation prevents MPTP opening, thereby preventing mitochondria swelling and changes in the membrane potential, and initiating the mitochondria-dependent cell death cascade.

\section{X-linked adrenoleukodystrophy as a model of neurodegenerative and non-neurodegen- erative diseases distinguished by alterations in redox homeostasis}

Oxidative stress, mitochondrial dysfunction and energetic failure are common to a number of ailments irrespective of the initial cause (Galea et al., 2012). It has been described how abnormally aggregated proteins act as molecular signals to induce CypD translocation to the inner mitochondrial membrane, resulting in mitochondrial dysfunction. $\mathrm{Du}$ and coworkers reported the existence of amyloid- $\beta$ (A $\beta$ )-CypD complexes in the mitochondria of cortical neurons in amyloid precursor protein-overexpressing mice, a model of Alzheimer's disease. These complexes promote ROS generation and CypD recruitment to the inner mitochondrial membrane, triggering the opening of the MPTP and subsequent cell death (Du et al., 2008). Considering the absence of protein aggregates in $\mathrm{X}$-ALD, we propose that molecular signals involved in the CypD translocation to the inner mitochondrial membrane are triggered by oxidative stress-induced CypD expression and the direct oxidation of CypD protein, instead of amyloid beta, the molecular signal involved in CypD 


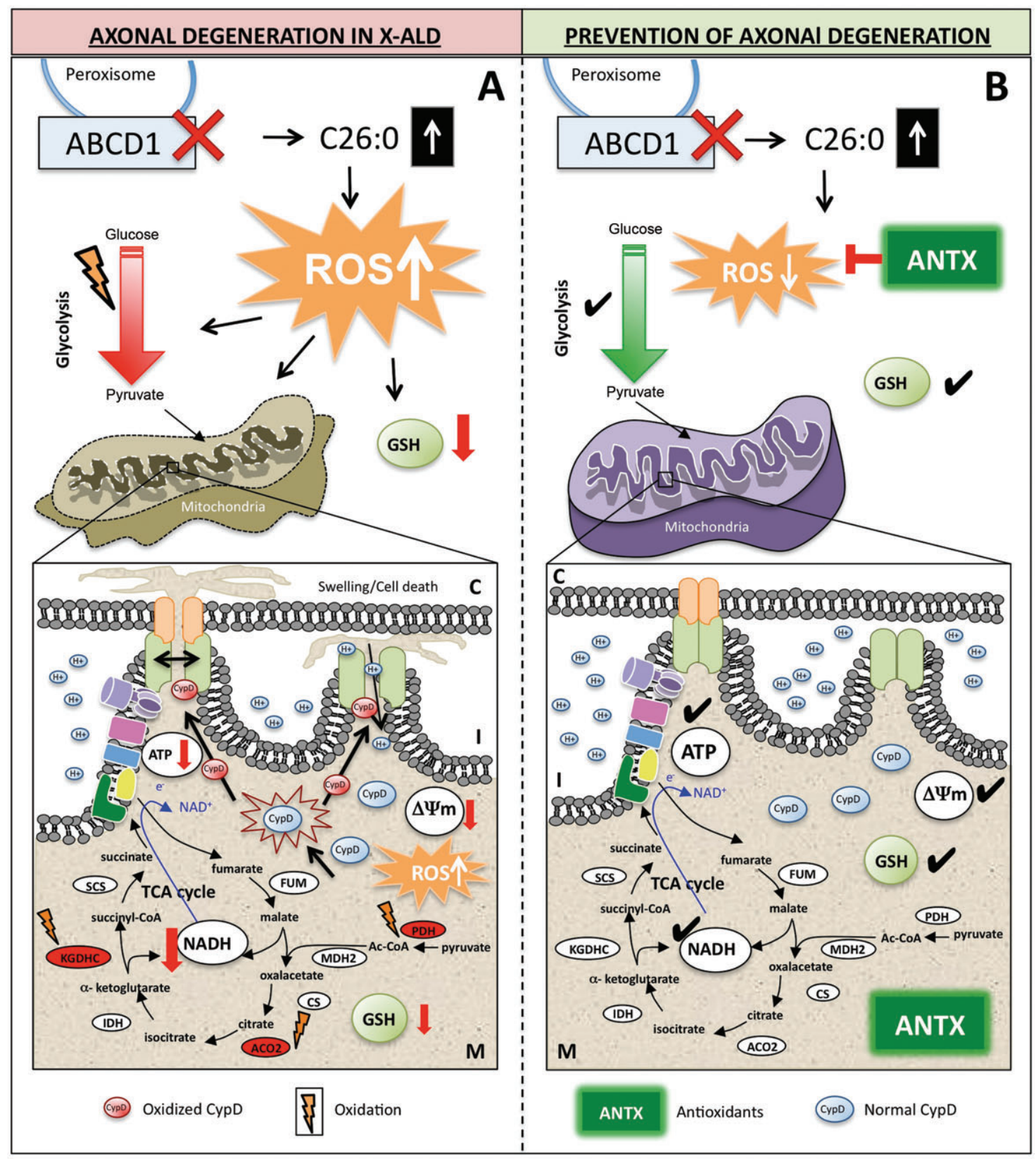

Figure 1 Working model for the molecular events underlying axonal degeneration and the action of antioxidants in the prevention of X-linked adrenoleukodystrophy.

(A) Mutations in the $A B C D 1$ gene produce C26:0 accumulation and reactive oxygen species (ROS) generation as a consequence. This affects glycolysis and the tricarboxylic acid cycle through the oxidation of the enzymes involved, thereby decreasing their activity. Hence, reduced levels of NADH and ATP lead to energetic deficiency. High levels of ROS consume glutathione levels, which become depleted. Further, ROS oxidize mitochondrial cyclophilin D inducing its translocation to the inner mitochondrial membrane and triggering the mitochondrial permeability transition pore opening. This results in inner mitochondrial membrane depolarization, mitochondrial swelling and subsequent cell death. (B) Antioxidant treatment decreases ROS levels, thus abrogating protein oxidation and preventing the impairment of glycolysis and the tricarboxylic acid cycle as well as the mitochondrial permeability transition pore opening. Likewise, lowered ROS content allows for the recovery of reduced glutathione levels and contributes to the maintenance of redox homeostasis. $\Delta \Psi \mathrm{m}$ : Inner mitochondrial membrane potential; C: cytosol; I: intermembrane space; M: matrix. 
translocation to the inner mitochondrial membrane in Alzheimer's disease.

\section{Antioxidants as a potential treatment against cyclophilin D-dependent diseases}

As shown in Figure 1B, antioxidants acting against C26:0induced ROS prevent enzyme oxidation, thus normalizing ATP and NADH content. Moreover, normalized GSH levels help to maintain redox homeostasis, preventing CypD oxidation and the subsequent mPTP opening. In addition to reversing oxidative lesions in CypD, antioxidants may contribute to the repair of already initiated CypD-dependent mitochondrial dysfunction. That is antioxidants could serve as MPTP opening inhibitors, which implies the rational use of these molecules for the treatment of CypDdependent diseases.

Received November 13, 2012; accepted March 2, 2013; previously published online March 14, 2013

\section{References}

Baarine, M., Andréoletti, P., Athias, A., Nury, T., Zarrouk, A., Ragot, K., Vejux, A., Riedinger, J.M., Kattan, Z., Bessede, G., et al. (2012a). Evidence of oxidative stress in very long chain fatty acid-treated oligodendrocytes and potentialization of ROS production using RNA interference-directed knockdown of ABCD1 and ACOX1 peroxisomal proteins. Neuroscience 213, 1-18.

Baarine, M., Ragot, K., Athias, A., Nury, T., Kattan, Z., Genin, E.C., Andreoletti, P., Ménétrier, F., Riedinger, J.M., Bardou, M., et al. (2012b). Incidence of Abcd1 level on the induction of cell death and organelle dysfunctions triggered by very long chain fatty acids and TNF-a on oligodendrocytes and astrocytes. Neurotoxicology 33, 212-228.

Baines, C.P., Kaiser, R.A., Purcell, N.H., Blair, N.S., Osinska, H., Hambleton, M.A., Brunskill, E.W., Sayen, M.R., Gottlieb, R.A., Dorn, G.W., et al. (2005). Loss of cyclophilin D reveals a critical role for mitochondrial permeability transition in cell death. Nature 434, 658-662.

Berger, J. and Gartner, J. (2006). X-linked adrenoleukodystrophy: clinical, biochemical and pathogenetic aspects. Biochim. Biophys. Acta 1763, 1721-1732.

Berger, J., Pujol, A., Aubourg, P., and Forss-Petter, S. (2010). Current and future pharmacological treatment strategies in X-linked adrenoleukodystrophy. Brain Pathol. 20, 845-856.

Bernardi, P., Krauskopf, A., Basso, E., Petronilli, V., Blachly-Dyson, E., Di Lisa, F., and Forte, M.A. (2006). The mitochondrial permeability transition from in vitro artifact to disease target. Febs J 273, 2077-2099.

Bezman, L., Moser, A.B., Raymond, G.V., Rinaldo, P., Watkins, P.A., Smith, K.D., Kass, N.E., and Moser, H.W. (2001). Adrenoleukodystrophy: incidence, new mutation rate, and results of extended family screening. Ann. Neurol. 49, 512-517.

Blackstone, C. and Chang, C.R. (2011). Mitochondria unite to survive. Nat Cell Biol 13, 521-522.

Connern, C.P. and Halestrap, A.P. (1994). Recruitment of mitochondrial cyclophilin to the mitochondrial inner membrane under conditions of oxidative stress that enhance the opening of a calcium-sensitive non-specific channel. Biochem. ). 302 (Pt 2), 321-324.

Crompton, M., Ellinger, H., and Costi, A. (1988). Inhibition by cyclosporin $\mathrm{A}$ of a $\mathrm{Ca}^{2+}$-dependent pore in heart mitochondria activated by inorganic phosphate and oxidative stress. Biochem. J. 255, 357-360.
Du, H., Guo, L., Fang, F., Chen, D., Sosunov, A.A., McKhann, G.M., Yan, Y., Wang, C., Zhang, H., Molkentin, J.D., et al. (2008). Cyclophilin D deficiency attenuates mitochondrial and neuronal perturbation and ameliorates learning and memory in Alzheimer's disease. Nat. Med. 14, 1097-1105.

Du, H. and Yan, S.S. (2010). Mitochondrial permeability transition pore in Alzheimer's disease: cyclophilin D and amyloid b. Biochim. Biophys. Acta 1802, 198-204.

Eichler, F.S., Ren, J.Q., Cossoy, M., Rietsch, A.M., Nagpal, S., Moser, A.B., Frosch, M.P., and Ransohoff, R.M. (2008). Is microglial apoptosis an early pathogenic change in cerebral X-linked adrenoleukodystrophy? Ann. Neurol. 63, 729-742.

Engelen, M., Kemp, S., de Visser, M., van Geel, B.M., Wanders, R.J., Aubourg, P., and Poll-The, B.T. (2012). X-linked adrenoleukodystrophy (X-ALD): clinical presentation and guidelines for diagnosis, follow-up and management. Orphanet. J. Rare Dis. 7, 51, 1-14.

Fernandez-Checa, J.C., Fernandez, A., Morales, A., Mari, M., Garcia-Ruiz, C., and Colell, A. (2010). Oxidative stress and altered mitochondrial function in neurodegenerative diseases: lessons from mouse models. CNS Neurol. Disord. Drug Targets 9, 439-454.

Ferrer, I., Aubourg, P., and Pujol, A. (2010). General aspects and neuropathology of $\mathrm{X}$-linked adrenoleukodystrophy. Brain Pathol. 20, 817-830.

Ferrer, I., Kapfhammer, J.P., Hindelang, C., Kemp, S., TrofferCharlier, N., Broccoli, V., Callyzot, N., Mooyer, P., Selhorst, J., Vreken, P., et al. (2005). Inactivation of the peroxisomal ABCD2 transporter in the mouse leads to late-onset ataxia involving mitochondria, Golgi and endoplasmic reticulum damage. Hum. Mol. Genet. 14, 3565-3577.

Forss-Petter, S., Werner, H., Berger, J., Lassmann, H., Molzer, B., Schwab, M.H., Bernheimer, H., Zimmermann, F., and Nave, K.A. (1997). Targeted inactivation of the X-linked adrenoleukodystrophy gene in mice. J. Neurosci. Res. 50, 829-843.

Forte, M., Gold, B.G., Marracci, G., Chaudhary, P., Basso, E., Johnsen, D., Yu, X., Fowlkes, J., Rahder, M., Stem, K., et al. (2007). Cyclophilin D inactivation protects axons in experimental autoimmune encephalomyelitis, an animal model of multiple sclerosis. Proc. Natl. Acad. Sci. USA 104, 7558-7563.

Fourcade, S., Lopez-Erauskin, J., Galino, J., Duval, C., Naudi, A., Jove, M., Kemp, S., Villarroya, F., Ferrer, I., Pamplona, R., et al. 
(2008). Early oxidative damage underlying neurodegeneration in X-adrenoleukodystrophy. Hum. Mol. Genet. 17, 1762-1773.

Fourcade, S., Ruiz, M., Camps, C., Schluter, A., Houten, S.M., Mooyer, P.A., Pàmpols, T., Dacremont, G., Wanders, R.J., Giròs, M., et al. (2009). A key role for the peroxisomal $A B C D 2$ transporter in fatty acid homeostasis. Am. J. Physiol. Endocrinol. Metab. 296, E211-E221.

Fourcade, S., Ruiz, M., Guilera, C., Hahnen, E., Brichta, L., Naudi, A., Portero-Otín, M., Dacremont, G., Cartier, N., Wanders, R., et al. (2010). Valproic acid induces antioxidant effects in X-linked adrenoleukodystrophy. Hum. Mol. Genet. 19, 2005-2014.

Galea, E., Launay, N., Portero-Otin, M., Ruiz, M., Pamplona, R., Aubourg, P., Ferrer, I., and Pujol, A. (2012). Oxidative stress underlying axonal degeneration in adrenoleukodystrophy: a paradigm for multifactorial neurodegenerative diseases? Biochim. Biophys. Acta 1822, 1475-1488.

Galino, J., Ruiz, M., Fourcade, S., Schluter, A., Lopez-Erauskin, J., Guilera, C., Jove, M., Naudi, A., García-Arumí, E., Andreu, A.L., et al. (2011). Oxidative damage compromises energy metabolism in the axonal degeneration mouse model of $x$-adrenoleukodystrophy. Antioxid. Redox Signal. 15, 2095-2107.

Gandhi, S., Wood-Kaczmar, A., Yao, Z., Plun-Favreau, H., Deas, E., Klupsch, K., Downward, J., Latchman, D.S., Tabrizi, S.J., Wood, N.W., et al. (2009). PINK1-associated Parkinson's disease is caused by neuronal vulnerability to calcium-induced cell death. Mol. Cell 33, 627-638.

Garashi, M., Belchis, D., and Suzuki, K. (1976). Brain gangliosides in adrenoleukodystrophy. J. Neurochem. 27, 327-328.

Halestrap, A. (2005). Biochemistry: a pore way to die. Nature 434, 578-579.

Halestrap, A.P. (2009). What is the mitochondrial permeability transition pore? J. Mol. Cell Cardiol. 46, 821-831.

Halestrap, A.P. and Davidson, A.M. (1990). Inhibition of $\mathrm{Ca}^{2+}$ induced large-amplitude swelling of liver and heart mitochondria by cyclosporin is probably caused by the inhibitor binding to mitochondrial-matrix peptidyl-prolyl cis-trans isomerase and preventing it interacting with the adenine nucleotide translocase. Biochem. J. 268, 153-160.

Hein, S., Schonfeld, P., Kahlert, S., and Reiser, G. (2008). Toxic effects of X-linked adrenoleukodystrophy-associated, very long chain fatty acids on glial cells and neurons from rat hippocampus in culture. Hum. Mol. Genet. 17, 1750-1761.

Hubbard, W.C., Moser, A.B., Tortorelli, S., Liu, A., Jones, D., and Moser, H. (2006). Combined liquid chromatographytandem mass spectrometry as an analytical method for high throughput screening for X-linked adrenoleukodystrophy and other peroxisomal disorders: preliminary findings. Mol. Genet. Metab. 89, 185-187.

Karlsson, J., Fong, K.S., Hansson, M.J., Elmer, E., Csiszar, K., and Keep, M.F. (2004). Life span extension and reduced neuronal death after weekly intraventricular cyclosporin injections in the G93A transgenic mouse model of amyotrophic lateral sclerosis. J. Neurosurg. 101, 128-137.

Kobayashi, T., Shinnoh, N., Kondo, A., and Yamada, T. (1997). Adrenoleukodystrophy protein-deficient mice represent abnormality of very long chain fatty acid metabolism. Biochem. Biophys. Res. Commun. 232, 631-636.

Laureti, S., Casucci, G., Santeusanio, F., Angeletti, G., Aubourg, P., and Brunetti, P. (1996). X-linked adrenoleukodystrophy is a frequent cause of idiopathic Addison's disease in young adult male patients. J. Clin. Endocrinol. Metab. 81, 470-474.
Lin, M.T. and Beal, M.F. (2006). Mitochondrial dysfunction and oxidative stress in neurodegenerative diseases. Nature 443, 787-795.

Linard, D., Kandlbinder, A., Degand, H., Morsomme, P., Dietz, K.J., and Knoops, B. (2009). Redox characterization of human cyclophilin D: identification of a new mammalian mitochondrial redox sensor? Arch. Biochem. Biophys. 491, 39-45.

Lopez-Erauskin, J., Fourcade, S., Galino, J., Ruiz, M., Schluter, A., Naudi, A., Jove, M., Portero-Otin, M., Pamplona, R., Ferrer, I., et al. (2011). Antioxidants halt axonal degeneration in a mouse model of X-adrenoleukodystrophy. Ann. Neurol. 70, 84-92.

Lopez-Erauskin, J., Galino, J., Bianchi, P., Fourcade, S., Andreu, A.L., Ferrer, I., Muñoz-Pinedo, C., and Pujol, A. (2012). Oxidative stress modulates mitochondrial failure and cyclophilin D function in X-linked adrenoleukodystrophy. Brain 135, 3584-3598.

Lu, J.F., Lawler, A.M., Watkins, P.A., Powers, J.M., Moser, A.B., Moser, H.W., and Smith, K.D. (1997). A mouse model for X-linked adrenoleukodystrophy. Proc. Natl. Acad. Sci. USA 94, 9366-9371.

Martin, L.J. (2010). The mitochondrial permeability transition pore: a molecular target for amyotrophic lateral sclerosis therapy. Biochim. Biophys. Acta 1802, 186-197.

Martin, L.J., Gertz, B., Pan, Y., Price, A.C., Molkentin, J.D., and Chang, Q. (2009). The mitochondrial permeability transition pore in motor neurons: involvement in the pathobiology of ALS mice. Exp. Neurol. 218, 333-346.

Martinez, A., Portero-Otin, M., Pamplona, R., and Ferrer, I. (2010). Protein targets of oxidative damage in human neurodegenerative diseases with abnormal protein aggregates. Brain Pathol. 20, 281-297.

Moser, H.W., Mahmood, A., and Raymond, G.V. (2007). X-linked adrenoleukodystrophy. Nat. Clin. Pract. Neurol. 3, 140-151.

Mosser, J., Douar, A.M., Sarde, C.O., Kioschis, P., Feil, R., Moser, H., Poustka, A.M., Mandel, J.L., and Aubourg, P. (1993). Putative $X$-linked adrenoleukodystrophy gene shares unexpected homology with ABC transporters. Nature 361, 726-730.

Nakagawa, T., Shimizu, S., Watanabe, T., Yamaguchi, O., Otsu, K., Yamagata, H., Inohara, H., Kubo, T., and Tsujimoto, Y. (2005). Cyclophilin D-dependent mitochondrial permeability transition regulates some necrotic but not apoptotic cell death. Nature 434, 652-658.

Naudi, A., Jove, M., Cacabelos, D., Ramirez, O., Ayala, V., Ilieva, E., Kichev, A., Ferrer, I., Portero-Otin, M., and Pamplona, R. (2011). Membrane unsaturation, selective neuronal vulnerability, and age-related neurodegenerative diseases. In: Lipid Peroxidation: Biological Implications. A. Catala, ed. (Argentina: Universidad Nacional de La Plata), pp. 47-70.

Nguyen, T.T., Stevens, M.V., Kohr, M., Steenbergen, C., Sack, M.N., and Murphy, E. (2011). Cysteine 203 of cyclophilin D is critical for cyclophilin D activation of the mitochondrial permeability transition pore. J. Biol. Chem. 286, 40184-40192.

Pamplona, R. and Barja, G. (2007). Highly resistant macromolecular components and low rate of generation of endogenous damage: two key traits of longevity. Ageing Res. Rev. 6, 189-210.

Peters, C., Charnas, L.R., Tan, Y., Ziegler, R.S., Shapiro, E.G., DeFor, T., Grewal, S.S., Orchard, P.J., Abel, S.L., Goldman, A.I., et al. (2004). Cerebral X-linked adrenoleukodystrophy: the international hematopoietic cell transplantation experience from 1982 to 1999. Blood 104, 881-888. 
Petronilli, V., Costantini, P., Scorrano, L., Colonna, R., Passamonti, S., and Bernardi, P. (1994). The voltage sensor of the mitochondrial permeability transition pore is tuned by the oxidation-reduction state of vicinal thiols. Increase of the gating potential by oxidants and its reversal by reducing agents. J. Biol. Chem. 269, 16638-16642.

Powers, J.M. and Moser, H.W. (1998). Peroxisomal disorders: genotype, phenotype, major neuropathologic lesions, and pathogenesis. Brain Pathol. 8, 101-120.

Powers, J.M., DeCiero, D.P., Ito, M., Moser, A.B., and Moser, H.W. (2000). Adrenomyeloneuropathy: a neuropathologic review featuring its noninflammatory myelopathy. J. Neuropathol. Exp. Neurol. 59, 89-102.

Powers, J.M., Pei, Z., Heinzer, A.K., Deering, R., Moser, A.B., Moser, H.W., Watkins, P.A., and Smith, K.D. (2005). Adreno-leukodystrophy: oxidative stress of mice and men. J. Neuropathol. Exp. Neurol. 64, 1067-1079.

Pratico, D. (2008). Evidence of oxidative stress in Alzheimer's disease brain and antioxidant therapy: lights and shadows. Ann. N Y Acad. Sci. 1147, 70-78.

Pujol, A., Hindelang, C., Callizot, N., Bartsch, U., Schachner, M., and Mandel, J.L. (2002). Late onset neurological phenotype of the $X$-ALD gene inactivation in mice: a mouse model for adrenomyeloneuropathy. Hum. Mol. Genet. 11, 499-505.

Pujol, A., Ferrer, I., Camps, C., Metzger, E., Hindelang, C., Callizot, N., Pàmpols, T., Giròs, M., and Mandel, J.L. (2004). Functional overlap between $A B C D 1$ (ALD) and ABCD2 (ALDR) transporters: a therapeutic target for X-adrenoleukodystrophy. Hum. Mol. Genet. 13, 2997-3006.

Schinzel, A.C., Takeuchi, O., Huang, Z., Fisher, J.K., Zhou, Z., Rubens, J., Hetz, C., Danial, N.N., Moskowitz, M.A., and Korsmeyer, S.J. (2005). Cyclophilin D is a component of mitochondrial permeability transition and mediates neuronal cell death after focal cerebral ischemia. Proc. Natl. Acad. Sci. USA 102, 12005-12010.

Schluter, A., Espinosa, L., Fourcade, S., Galino, J., Lopez, E., Ilieva, E., Morató, L., Asheuer, M., Cook, T., McLaren, A., et al. (2012). Functional genomic analysis unravels a metabolicinflammatory interplay in adrenoleukodystrophy. Hum. Mol. Genet. 21, 1062-1077.

Schonbrunner, E.R., Mayer, S., Tropschug, M., Fischer, G., Takahashi, N., and Schmid, F.X. (1991) Catalysis of protein folding by cyclophilins from different species. J. Biol. Chem. 266, 3630-3635.

Shapiro, E., Krivit, W., Lockman, L., Jambaque, I., Peters, C., Cowan, M., Blanche, S., Bordigoni, P., Loes, D., Ziegler, R., et al. (2000). Long-term effect of bone-marrow transplantation for childhood-onset cerebral X-linked adrenoleukodystrophy. Lancet 356, 713-718.

Shirendeb, U., Reddy, A.P., Manczak, M., Calkins, M.J., Mao, P., Tagle, D.A., and Reddy, P.H. (2011). Abnormal mitochondrial dynamics, mitochondrial loss and mutant huntingtin oligomers in Huntington's disease: implications for selective neuronal damage. Hum. Mol. Genet. 20, 1438-1455.

Singh, I., Moser, A.E., Moser, H.W., and Kishimoto, Y. (1984). Adrenoleukodystrophy: impaired oxidation of very long chain fatty acids in white blood cells, cultured skin fibroblasts, and amniocytes. Pediatr. Res. 18, 286-290.

Singh, I. and Pujol, A. (2010). Pathomechanisms underlying $X$-adrenoleukodystrophy: a three-hit hypothesis. Brain Pathol. 20, 838-844.

Stack, E.C., Matson, W.R., and Ferrante, R.J. (2008). Evidence of oxidant damage in Huntington's disease: translational strategies using antioxidants. Ann. N Y Acad. Sci. 1147, 79-92.

Thannickal, V.J. and Fanburg, B.L. (2000). Reactive oxygen species in cell signaling. Am J. Physiol. Lung. Cell Mol. Physiol. 279, L1005-L1028.

Thomas, B., Banerjee, R., Starkova, N.N., Zhang, S.F., Calingasan, N.Y., Yang, L., Wille, E., Lorenzo, B.J., Ho, D.J., Beal, M.F., et al. (2011). Mitochondrial permeability transition pore component cyclophilin D distinguishes nigrostriatal dopaminergic death paradigms in the MPTP mouse model of Parkinson's disease. Antioxid. Redox Signal. 16, 855-868.

van Roermund, C.W., Visser, W.F., Ijlst, L., van Cruchten, A., Boek, M., Kulik, W., Waterham, H.R., and Wanders, R.J. (2008). The human peroxisomal $A B C$ half transporter ALDP functions as a homodimer and accepts acyl-CoA esters. FASEB J. 22, 4201-4208.

Vargas, C.R., Wajner, M., Sirtori, L.R., Goulart, L., Chiochetta, M., Coelho, D., Latini, A., Llesuy, S., Bello-Klein, A., Giugliani, R., et al. (2004). Evidence that oxidative stress is increased in patients with X-linked adrenoleukodystrophy. Biochim. Biophys. Acta 1688, 26-32.

Wanders R.J., van Roermund C.W., van Wijland M.J., Nijenhuis A.A., Tromp A., Schutgens R.B., Brouwer-Kelder, E.M., Schram, A.W., Tager, J.M., van den Bosch, H., et al. (1987). X-linked adrenoleukodystrophy: defective peroxisomal oxidation of very long chain fatty acids but not of very long chain fatty acyl-CoA esters. Clin. Chim. Acta 165, 321-329.

Wang, Y., Yang, J., and Yi, J. (2012). Redox sensing by proteins: oxidative modifications on cysteines and the consequent events. Antioxid. Redox Signal. 16, 649-657.

Zarrouk, A., Vejux, A., Nury, T., El Hajj, H.I., Haddad, M., Cherkaoui-Malki, M., Riedinger, J.M., Hammami, M., and Lizard, G. (2012). Induction of mitochondrial changes associated with oxidative stress on very long chain fatty acids (C22:0, C24:0, or C26:0)-treated human neuronal cells (SK-NB-E). Oxid. Med. Cell Longev. 2012, 623257.

Zhou, C., Huang, Y., and Przedborski, S. (2008). Oxidative stress in Parkinson's disease: a mechanism of pathogenic and therapeutic significance. Ann. N Y Acad. Sci. 1147, 93-104. 